\title{
Reduced Cardiovascular Capacity and Resting Metabolic Rate in Men with Prostate Cancer Undergoing Androgen Deprivation: A Comprehensive Cross-Sectional Investigation
}

\author{
Bradley A. Wall, ${ }^{1,2}$ Daniel A. Galvão, ${ }^{2}$ Naeem Fatehee, ${ }^{2}$ Dennis R. Taaffe, ${ }^{2,3}$ Nigel Spry, $, 4,5$ \\ David Joseph, ${ }^{2,4,5}$ and Robert U. Newton ${ }^{2,6}$ \\ ${ }^{1}$ School of Psychology and Exercise Science, Murdoch University, Murdoch, WA 6150, Australia \\ ${ }^{2}$ Exercise Medicine Research Institute, Edith Cowan University, Joondalup, WA 6027, Australia \\ ${ }^{3}$ School of Medicine, University of Wollongong, Wollongong, NSW 2522, Australia \\ ${ }^{4}$ Department of Radiation Oncology, Sir Charles Gairdner Hospital, Nedlands, WA 6009, Australia \\ ${ }^{5}$ Faculty of Medicine, University of Western Australia, Nedlands, WA 6009, Australia \\ ${ }^{6}$ Centre for Clinical Research, The University of Queensland, Herston, QLD 4006, Australia
}

Correspondence should be addressed to Bradley A. Wall; b.wall@murdoch.edu.au

Received 31 July 2015; Accepted 5 October 2015

Academic Editor: In Ho Chang

Copyright (C) 2015 Bradley A. Wall et al. This is an open access article distributed under the Creative Commons Attribution License, which permits unrestricted use, distribution, and reproduction in any medium, provided the original work is properly cited.

\begin{abstract}
Objectives. To investigate if androgen deprivation therapy exposure is associated with additional risk factors for cardiovascular disease and metabolic treatment-related toxicities. Methods. One hundred and seven men (42-89 years) with prostate cancer undergoing androgen deprivation therapy completed a maximal graded objective exercise test to determine maximal oxygen uptake, assessments for resting metabolic rate, body composition, blood pressure and arterial stiffness, and blood biomarker analysis. A cross-sectional analysis was undertaken to investigate the potential impact of therapy exposure with participants stratified into two groups according to duration of androgen deprivation therapy ( $<3$ months and $\geq 3$ months). Results. Maximal oxygen uptake $(26.1 \pm 6.0 \mathrm{~mL} / \mathrm{kg} / \mathrm{min}$ versus $23.2 \pm 5.8 \mathrm{~mL} / \mathrm{kg} / \mathrm{min}, p=0.020)$ and resting metabolic rate $(1795 \pm 256 \mathrm{kcal} / \mathrm{d}$ versus $1647 \pm 236 \mathrm{kcal} / \mathrm{d}, p=0.005)$ were significantly higher in those with shorter exposure to androgen deprivation. There were no differences between groups for peripheral and central blood pressure, arterial stiffness, or metabolic profile. Conclusion. Three months or longer exposure to androgen deprivation therapy was associated with reduced cardiorespiratory capacity and resting metabolic rate, but not in a range of blood biomarkers. These findings suggest that prolonged exposure to androgen deprivation therapy is associated with negative alterations in cardiovascular outcomes. Trial registry is: ACTRN12609000200280.
\end{abstract}

\section{Introduction}

Androgen deprivation therapy (ADT) is a commonly used treatment for prostate cancer [1]. Although ADT improves prostate cancer survival, a number of studies have reported associations between ADT and treatment-related toxicities, such as increased risk of cardiovascular disease (CVD) and metabolic complications which can compromise survival and quality of life [2-4]. Moreover, several toxicities have been reported to be present soon after initiation of treatment. For example, increased arterial stiffness and adverse body compositional changes were associated with increasing insulin concentrations after 6 months of ADT treatment suggestive of impaired insulin sensitivity [5], and others have reported increases in serum insulin levels after only 3 months of ADT exposure [6]. ADT-related decline in physical function, grip strength, and self-reported physical function has been previously reported by Alibhai et al. [7] when compared with non-ADT controls; ADT users also had worse role physical function, bodily pain, and vitality. Importantly, these reductions in objective and self-reported measures of physical function were apparent within the first 3 months of initiating $\mathrm{ADT}$ treatment and persisted for at least 12 months. 
Metabolic implications of long-term ADT have been previously explored by Basaria et al. [3], who found men receiving $>12$ months ADT developed insulin resistance and hyperglycaemia, independent of age and body mass index (BMI). In addition, long-term ADT has been associated with diabetes and metabolic syndrome [8]. Keating et al. [9] were the first to report higher risks of cardiovascular and metabolic complications as well as sudden cardiac death in prostate cancer patients receiving ADT, whilst short-term ADT treatment was significantly associated with greater risks of disease and the elevated risks persisted in men on longer duration therapy [9]. Given the association between cardiorespiratory fitness and CVD mortality [10], the measurement of aerobic capacity can significantly improve the risk classification for CVD mortality [11]. While previous research has predominantly used surrogate measures of cardiorespiratory fitness such as the six-minute walk test (6MWT) or $400 \mathrm{~m}$ walk [7, 12], directly assessing aerobic capacity in ADT-treated men will further our understanding of the cardiovascular risks associated with this form of treatment. In this study, we report for the first time potential differences in relation to patients ADT exposure using objective measures of aerobic capacity, resting metabolic rate, blood pressure, arterial stiffness, and blood biomarkers. We hypothesized that longer-term ADT would lead to compromised aerobic capacity and metabolic parameters hence posing a greater risk for the development and progression of cardiovascular and metabolic diseases.

\section{Methods}

2.1. Participants. 272 patients with prostate cancer were screened for participation in a 12-month exercise trial [13] from February 2009 to August 2011 in Perth, Western Australia, and Brisbane, Queensland. 109 patients declined participation or were excluded for the following main reasons: declined to participate, too far to travel, unable to fit in with work, unable to obtain general practitioner/physician consent, and bone metastatic disease. In this report, we present the results from baseline assessment of a subgroup of 107 patients who undertook testing including cardiorespiratory capacity by October 2010 . This study was approved by the University Human Research Ethics Committee and all participants provided signed informed consent.

All participants underwent assessment for cardiorespiratory capacity, resting metabolic rate, peripheral and central blood pressure and arterial stiffness, and markers of metabolic health.

2.2. Cardiorespiratory Capacity. Participants performed a standardised progressive maximal walking test (Bruce Protocol) on a motorized treadmill supervised by a physician. Expired respiratory gases were collected (Parvo Metabolic Measuring System, Sandy, UT, USA) to determine maximal oxygen uptake (the maximal amount of oxygen that can be consumed and utilised, $\left.\mathrm{VO}_{2 \max }\right)$. A plateau in oxygen consumption was used as the criterion for achieving $\mathrm{VO}_{2 \max }$; if no plateau occurred, then a respiratory exchange ratio (RER) of $\geq 1.1$ was used. If the subject achieved no plateau in oxygen consumption or a RER value $<1.1$ their data were excluded. This direct assessment of peak oxygen consumption is considered the gold standard outcome of cardiorespiratory fitness or aerobic capacity [14]. The coefficient of variation for repeated maximal exercise tests is approximately $4 \%$ [15]. Blood pressure was measured during the last minute of each 3-minute stage via manual auscultatory technique.

2.3. Resting Metabolic Rate. Resting metabolic rate (RMR) was measured via respiratory gas analysis over 20 minutes. A 5 -minute period that showed an oxygen consumption with a coefficient of variation of $<10 \%$ was selected for analysis [16]. The coefficient of variation for RMR is $<3 \%$.

2.4. Resting Blood Pressure and Arterial Stiffness. Brachial blood pressure was recorded at the dominant arm in triplicate via a validated oscillometric device (HEM-705CP, Omron Corporation, Japan) [17]. Applanation tonometry (SPC-301, Millar Instruments, Houston, Texas, USA) was used to measure radial artery pressure waveforms at the right arm. A generalised transfer function was applied to obtain the central pressure waveform at the ascending aorta. Pulse wave analysis was used to determine central blood pressure and indices of arterial stiffness, performed using SphygmoCor version 6.1 software (AtCor Medical, Sydney, Australia). Assessing central blood pressure using this method has been validated against invasive techniques [18]. The augmentation index (AIx) refers to the ratio of augmentation to central pulse pressure, expressed as a percentage, and measures systemic arterial stiffness. Variability has been previously reported as $0.3 \mathrm{mmHg}$ for central systolic pressure and $1.5 \%$ for the AIx. Carotid-to-radial pulse wave velocity was measured by collecting arterial pressure waves at both the carotid and radial locations. The reported coefficient of variation for forearm (radial) pulse wave velocity is $2.9 \%$ whilst the brachial pulse wave velocity coefficient of variation is $7.7 \%$ [19].

2.5. Metabolic Syndrome. Patients were classified as having metabolic syndrome if they met three of the following five criteria according to the Adult Panel III Criteria [20]: (1) plasma glucose level more than $110 \mathrm{mg} / \mathrm{dL}$, (2) serum triglyceride levels $\geq 150 \mathrm{mg} / \mathrm{dL}$, (3) serum high density lipoprotein less than $40 \mathrm{mg} / \mathrm{dL}$, (4) waist circumference greater than $102 \mathrm{~cm}$, and (5) blood pressure $\geq 135 / 80 \mathrm{mmHg}$. Patients on antihypertensive and antilipid medications were also considered positive for the respective criterion.

2.6. Other Measures. Venous blood samples $(2 \times 8.5 \mathrm{~mL})$ were obtained from the antecubital vein with whole blood analysed for haemoglobin A1C (HbA1C, \%) whilst the remaining blood was separated and analysed for testosterone, insulin, prostate specific antigen, triglycerides, LDL cholesterol, HDL cholesterol, total cholesterol, glucose, and C-reactive protein. All blood variables were analysed commercially by accredited Australian National Association of Testing Authorities laboratories (Pathwest Laboratory Medicine, WA).

Whole body bone mineral-free lean mass and fat mass, trunk fat, and body fat percentage were assessed by dual 
TABLE 1: Subject characteristics for the short- and longer-term androgen deprivation groups.

\begin{tabular}{|c|c|c|c|c|}
\hline Variable & $\begin{array}{c}<3 \text { months } \\
\text { Mean } \pm \text { SD } \\
n=57\end{array}$ & $\begin{array}{c}\geq 3 \text { months } \\
\text { Mean } \pm \text { SD } \\
\quad N=50\end{array}$ & Mean difference (95\% CI) & $p$ value \\
\hline Age (years) & $67.6 \pm 8.9$ & $69.9 \pm 9.7$ & $-2.3(-5.9,1.3)$ & 0.395 \\
\hline Prostate specific antigen $\left(\mathrm{ng} \cdot \mathrm{mL}^{-1}\right)$ & $1.2 \pm 1.7$ & $1.4 \pm 2.6$ & $-0.2(-1.1,0.7)$ & 0.659 \\
\hline Gleason Score & $7.6 \pm 0.8$ & $7.7 \pm 1.4$ & $-0.1(-0.6,0.6)$ & 0.985 \\
\hline Testosterone $\left(\mathrm{pg} \cdot \mathrm{mL}^{-1}\right)$ & $1.3 \pm 1.5$ & $1.5 \pm 3.4$ & $-0.2(-1.2,0.8)$ & 0.651 \\
\hline Height $(\mathrm{cm})$ & $172.2 \pm 6.4$ & $172.7 \pm 6.3$ & $-0.5(-3.2,2.1)$ & 0.648 \\
\hline Body mass $(\mathrm{kg})$ & $85.7 \pm 13.6$ & $83.4 \pm 14.0$ & $2.3(-3.1,7.8)$ & 0.105 \\
\hline Body mass index $\left(\mathrm{kg} \cdot \mathrm{m}^{2}\right)$ & $28.9 \pm 4.3$ & $28.0 \pm 3.9$ & $0.9(-0.7,2.4)$ & 0.215 \\
\hline Waist circumference $(\mathrm{cm})$ & $99.2 \pm 12.1$ & $100.3 \pm 12.9$ & $-0.9(-5.5,3.9)$ & 0.737 \\
\hline
\end{tabular}

energy X-ray absorptiometry (DEXA, Hologic Discovery A, Waltham, MA, USA). In addition, appendicular lean mass was calculated as the sum of upper and lower limb lean mass. The coefficient of variation for body composition measures is $<1 \%$.

2.7. Statistical Analysis. Sample size calculations for the initial RCT [13] resulted in a requirement for 65 subjects per group at the commencement of the study. For the principal analyses in this report, we had $80 \%$ power to detect a significant difference in METS and similarly for $\mathrm{VO}_{2 \max }$ in absolute $(\mathrm{L} / \mathrm{min})$ and relative terms $(\mathrm{mL} / \mathrm{kg} / \mathrm{min})$, and $87 \%$ for RMR in $\mathrm{kcal} / 24 \mathrm{hr}$.

Analyses were performed using the Statistical Package for Social Sciences version 18.0 software (PASW, Chicago, IL, USA). Normality of the data was assessed using the Kolmogorov-Smirnov test. The analyses included standard descriptive statistics, Student's independent $t$-tests, and Pearson's chi-square test for categorical variables. Potential differences between patients on $<3$ months or $\geq 3$ months on ADT were undertaken based on the previous prospective work by Alibhai and colleagues [7] which showed that even shortterm treatment leads to substantial deterioration in physical function. All tests were two-tailed and an alpha level of 0.05 was applied as the criterion for statistical significance. Results are reported as the mean \pm standard deviation.

\section{Results}

3.1. Subject Characteristics. Characteristics for all participants were age $68.6 \pm 8.8 \mathrm{~kg}$; height $172.4 \pm 6.3 \mathrm{~cm}$; and body mass $84.0 \pm 13.7 \mathrm{~kg}$. Mean ADT duration was $2.0 \pm 0.0$ and $7.1 \pm 6.2$ months for the shorter and longer groups, respectively. There were no significant differences in any subject characteristics (Table 1) or lean and fat mass (Table 2) based on ADT exposure.

3.2. Cardiorespiratory Capacity. 91 (85\%) of the participants were able to achieve the desired criteria for $\mathrm{VO}_{2 \max }$ with no difference between groups in their ability to achieve $\mathrm{VO}_{2 \max }$. Maximal oxygen consumption was significantly higher in the shorter ADT duration group when presented

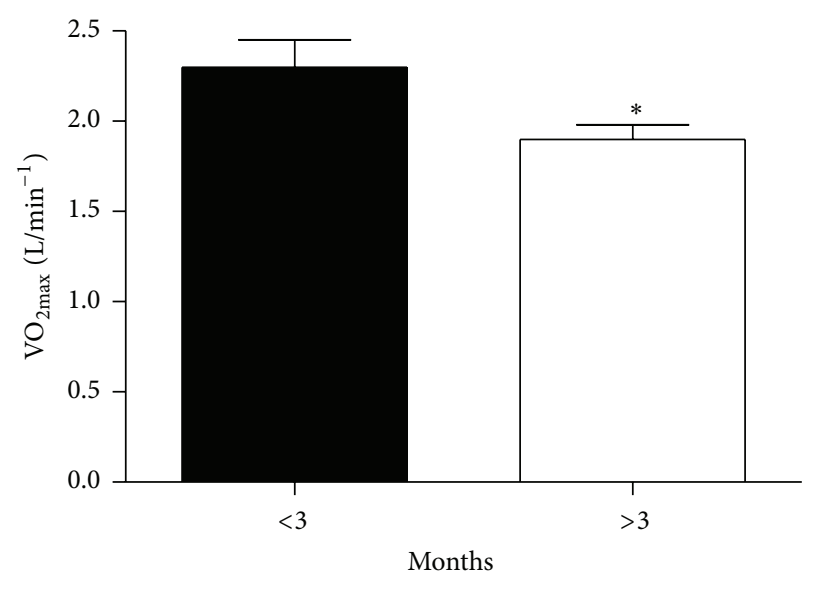

FIGURE 1: Absolute $\mathrm{VO}_{2 \max }$ values (mean $\pm \mathrm{SE}$ ) for the short- and longer-term androgen deprivation groups. $*$ denotes significant difference versus $<3$ months.

in absolute $\left(\mathrm{L} / \mathrm{min}^{-1}\right)(p=0.035)$ (Figure 1) or relative terms $\left(\mathrm{mL} / \mathrm{kg} / \mathrm{min}^{-1}\right)(p=0.020)$ (Table 3$)$. Corresponding metabolic equivalents were also significantly higher in the shorter duration ADT group $(p=0.02)$. Whilst test duration was not statistically significant, shorter duration group exhibited an additional 54 seconds $(p=0.080)$ of walking endurance.

3.3. Resting Metabolic Rate. There was a significant difference observed in RMR with the shorter duration group recording a significantly higher $(p=0.005) \mathrm{RMR}$ in absolute terms and relative to body mass $\mathrm{RMR}(p=0.017)$ compared to the longer duration group (Table 3 ). Whilst not statistically significant $(p=0.079)$, RMR relative to lean body mass was $1.3 \mathrm{kcal} / \mathrm{lean} \mathrm{kg} / 24 \mathrm{hr}$ higher in those with shorter ADT exposure.

3.4. Central Blood Pressure. There were no differences between short and longer ADT exposure in any of the central or peripheral blood pressure variables or central augmentation index (Table 3). Further, there was no difference in pulse wave velocity between groups (Table 3 ). 
TABLE 2: Body composition and blood markers for the short- and longer-term androgen deprivation groups.

\begin{tabular}{|c|c|c|c|c|}
\hline Variable & $\begin{array}{c}<3 \text { months } \\
\text { Mean } \pm \text { SD } \\
n=57\end{array}$ & $\begin{array}{c}\geq 3 \text { months } \\
\text { Mean } \pm \mathrm{SD} \\
\quad N=50\end{array}$ & Mean difference $(95 \% \mathrm{CI})$ & $p$ value \\
\hline \multicolumn{5}{|l|}{ Lean tissue mass (kg) } \\
\hline Whole body & $60.1 \pm 7.5$ & $58.0 \pm 8.6$ & $2.1(-1.0,5.2)$ & 0.184 \\
\hline Appendicular & $25.6 \pm 3.3$ & $24.5 \pm 3.9$ & $1.1(-0.3,2.5)$ & 0.112 \\
\hline \multicolumn{5}{|l|}{ Fat mass $(\mathrm{kg})$} \\
\hline Whole body & $23.1 \pm 7.3$ & $24.6 \pm 8.3$ & $-1.5(-4.5,1.4)$ & 0.307 \\
\hline Trunk & $12.4 \pm 4.5$ & $12.9 \pm 5.8$ & $-0.5(-2.5,1.5)$ & 0.627 \\
\hline Body fat $\%$ & $26.4 \pm 5.1$ & $28.3 \pm 5.2$ & $-1.9(-4.0,0.1)$ & 0.053 \\
\hline \multicolumn{5}{|l|}{ Blood markers } \\
\hline HbA1C (\%) & $6.4 \pm 3.7$ & $6.1 \pm 1.0$ & $0.3(-0.8,1.3)$ & 0.638 \\
\hline Testosterone $\left(\mathrm{pg} \cdot \mathrm{mL}^{-1}\right)$ & $1.3 \pm 1.5$ & $1.5 \pm 3.4$ & $-0.2(-1.2,0.8)$ & 0.651 \\
\hline Prostate specific antigen $\left(\mathrm{ng} \cdot \mathrm{mL}^{-1}\right)$ & $1.2 \pm 1.7$ & $1.4 \pm 2.6$ & $-0.2(-1.1,0.7)$ & 0.659 \\
\hline Insulin $(\mathrm{mU} / \mathrm{L})$ & $11.2 \pm 6.7$ & $9.2 \pm 4.3$ & $2.0(-0.3,4.2)$ & 0.085 \\
\hline Triglycerides $(\mathrm{mmol} / \mathrm{L})$ & $1.3 \pm 0.6$ & $1.4 \pm 0.7$ & $-0.1(-0.3,0.1)$ & 0.393 \\
\hline LDL cholesterol (mmol/L) & $2.8 \pm 0.9$ & $2.9 \pm 1.0$ & $-0.1(-0.5,0.3)$ & 0.575 \\
\hline HDL cholesterol (mmol/L) & $1.3 \pm 0.4$ & $1.4 \pm 0.4$ & $-0.1(-0.3,0.1)$ & 0.269 \\
\hline Total cholesterol (mmol/L) & $4.7 \pm 1.1$ & $4.9 \pm 1.0$ & $-0.2(-0.6,0.2)$ & 0.287 \\
\hline Glucose $(\mathrm{mmol} / \mathrm{L})$ & $5.5 \pm 1.1$ & $5.9 \pm 1.9$ & $-0.4(-1.0,0.2)$ & 0.216 \\
\hline C-reactive protein $(\mathrm{mg} / \mathrm{L})$ & $2.9 \pm 3.3$ & $2.5 \pm 2.0$ & $0.4(-0.7,1.5)$ & 0.469 \\
\hline
\end{tabular}

HbA1C: glycated haemoglobin; LDL: low density lipoprotein; HDL: high density lipoprotein.

3.5. Metabolic Profile. No significant differences were observed between short and longer ADT exposure in any of the blood markers analysed (Table 2). Whilst not statistically significant, insulin was $19.6 \%$ higher in the shorter duration group $(p=0.085)$.

3.6. Metabolic Syndrome Variables. According to National Cholesterol Education Program Adult Treatment Panel III, $20.3 \%$ of the acute group and $13.5 \%$ of the chronic group were classified as having metabolic syndrome $(p=0.337)$. However, there were no significant differences observed between groups for fasting plasma glucose $(p=0.998)$, serum triglycerides $(p=0.874)$, serum HDL $(p=0.815)$, waist circumference $(p=0.994)$, or hypertension $(p=0.093)$.

\section{Discussion}

We examined the difference between patients on either shortor longer-term ADT across a variety of cardiovascular and metabolic parameters to determine if additional therapy time exposure is associated with accumulating CVD risk factors or metabolic treatment-related toxicities. Those exposed to ADT for a longer period of time had a lower cardiorespiratory capacity and RMR suggesting that longer ADT duration is associated with decline in cardiovascular capacity and aspects of metabolic function.

The maximal aerobic exercise testing protocol used in this study has been shown to be safe and feasible in this population [21]. To our knowledge, this is the first research study to directly measure $\mathrm{VO}_{2 \max }$ in men on ADT to investigate the effect duration of treatment has on cardiorespiratory capacity. Our findings demonstrate that there are differences in cardiorespiratory capacity between men on shorter- and longer-term ADT. The $\mathrm{VO}_{2 \max }$ of the shorter duration ADT group was $21 \%$ higher than the longer exposed group, which has important implications when considering that the low levels of cardiorespiratory fitness have been associated with a markedly increased risk of premature death from all causes and in particular CVD in all populations including healthy older men and those with established cardiovascular disease [10]. Conversely, an increase in cardiorespiratory fitness is associated with a reduced risk of CVD [22]. No differences were observed in any of the blood pressure parameters in relation to ADT exposure.

ADT exposure time appears to influence resting energy expenditure with those men on longer duration ADT having a lower RMR. Fat-free mass plays a major role in the variance of RMR amongst individuals [23], with more recent research suggesting that both fat-free mass and fat mass significantly influence RMR [24]. Whilst no significant differences in any body composition values were reported, it is likely that the combined effects of the nonsignificant differences in lean body mass $(2 \mathrm{~kg})$ and fat mass $(1.5 \mathrm{~kg})$ contributed to this significant reduction in resting energy expenditure in the longer ADT exposure group. A reduced RMR with continuing ADT exposure would contribute to the accumulation of adipose tissue if dietary intake remained unchanged.

When exploring additional CVD risk factors, metabolic syndrome has been widely used as a surrogate marker for 
TABLE 3: Resting and maximal cardiorespiratory values and hemodynamic and pulse wave analysis parameters for the short- and longer-term androgen deprivation groups.

\begin{tabular}{|c|c|c|c|c|}
\hline Variable & $\begin{array}{c}<3 \text { months } \\
\text { Mean } \pm \text { SD } \\
n=57\end{array}$ & $\begin{array}{l}\geq 3 \text { months } \\
\text { Mean } \pm \text { SD } \\
N=50\end{array}$ & Mean difference $(95 \% \mathrm{CI})$ & $p$ value \\
\hline $\mathrm{VO}_{2 \max }\left(\mathrm{L} / \mathrm{min}^{-1}\right)^{*}$ & $2.3 \pm 1.0$ & $1.9 \pm 0.6$ & $0.4(0.1,0.7)$ & 0.035 \\
\hline $\mathrm{VO}_{2 \max }\left(\mathrm{mL} / \mathrm{kg} / \mathrm{min}^{-1}\right)^{*}$ & $26.1 \pm 6.0$ & $23.2 \pm 5.8$ & $2.9(0.5,5.3)$ & 0.020 \\
\hline $\mathrm{VO}_{2 \max }(\mathrm{METS})^{*}$ & $7.5 \pm 1.7$ & $6.6 \pm 1.6$ & $0.9(0.1,1.5)$ & 0.020 \\
\hline Test duration (mins) & $8.5 \pm 2.8$ & $7.6 \pm 2.6$ & $0.9(-0.1,2.1)$ & 0.080 \\
\hline $\begin{array}{l}\text { Resting metabolic rate } \\
(\mathrm{kcal} / 24 \mathrm{hr})^{*}\end{array}$ & $1795 \pm 256$ & $1647 \pm 236$ & $147(46,249)$ & 0.005 \\
\hline $\begin{array}{l}\text { Relative total body mass } \\
\text { Resting metabolic rate } \\
(\mathrm{kcal} / \mathrm{kg} / 24 \mathrm{hr})^{*}\end{array}$ & $21.5 \pm 3.0$ & $20.0 \pm 2.6$ & $1.5(0.3,2.7)$ & 0.017 \\
\hline $\begin{array}{l}\text { Relative lean body mass } \\
\text { Resting metabolic rate } \\
\text { (kcal/lean } \mathrm{kg} / 24 \mathrm{hr} \text { ) }\end{array}$ & $30.5 \pm 3.2$ & $29.2 \pm 4.1$ & $1.3(-0.2,2.9)$ & 0.079 \\
\hline Peripheral SBP (mmHg) & $150.9 \pm 19.9$ & $149.0 \pm 19.4$ & $1.9(-5.8,9.6)$ & 0.624 \\
\hline Peripheral DBP (mmHg) & $85.6 \pm 12.1$ & $84.5 \pm 10.4$ & $1.1(-3.4,5.6)$ & 0.626 \\
\hline Peripheral MAP (mmHg) & $108.4 \pm 15.0$ & $107.2 \pm 12.8$ & $1.2(-4.3,6.7)$ & 0.669 \\
\hline Central SBP (mmHg) & $139.1 \pm 21.1$ & $138.7 \pm 20.2$ & $0.4(-7.7,8.5)$ & 0.922 \\
\hline Central DBP (mmHg) & $86.8 \pm 12.3$ & $85.5 \pm 10.6$ & $1.3(-3.2,5.8)$ & 0.571 \\
\hline Central MAP (mmHg) & $108.9 \pm 16.1$ & $107.2 \pm 12.8$ & $1.7(-4.1,7.5)$ & 0.557 \\
\hline $\begin{array}{l}\text { Peripheral augmentation } \\
\text { index (AIx, \%) }\end{array}$ & $83.5 \pm 13.0$ & $86.5 \pm 13.9$ & $-3.0(-8.3,2.3)$ & 0.261 \\
\hline $\begin{array}{l}\text { Central augmentation } \\
\text { pressure }(\mathrm{mmHg})\end{array}$ & $15.4 \pm 8.2$ & $16.7 \pm 9.6$ & $-1.3(4.8,2.1)$ & 0.445 \\
\hline $\begin{array}{l}\text { Augmentation load } \\
(\mathrm{mmHg})\end{array}$ & $14.3 \pm 5.0$ & $14.6 \pm 4.9$ & $-0.3(-2.3,1.6)$ & 0.732 \\
\hline $\begin{array}{l}\text { Central augmentation } \\
\text { index (AIx, \%) }\end{array}$ & $140.4 \pm 17.8$ & $144.4 \pm 19.6$ & $-4.0(-11.3,3.3)$ & 0.281 \\
\hline Pulse wave velocity $(\mathrm{m} / \mathrm{s})$ & $10.0 \pm 1.4$ & $10.0 \pm 1.8$ & $0.0(-0.6,0.7)$ & 0.983 \\
\hline
\end{tabular}

$\mathrm{VO}_{2 \max }$ : maximal oxygen uptake; METS: metabolic equivalents; SBP: systolic blood pressure; DBP: diastolic blood pressure; MAP: mean arterial pressure; AIx: augmentation index.

${ }^{*}$ refers to a significant difference between $<3$ months and $\geq 3$ months.

CVD. In our study, $20.3 \%$ and $13.5 \%$ of the shorter and longer ADT exposure groups, respectively, met the criteria for metabolic syndrome. These values are lower than the 55\% of ADT-treated prostate cancer patients previously reported by Braga-Basaria et al. [25] but similar to non-ADT-treated prostate cancer patients (22\%) and control subjects $(20 \%)$ reported in the same study [25]. Waist circumference and hypertension appear to be the two most common cardiovascular risk factors present in both groups, demonstrating that these risk factors are present irrespective of the treatment duration.

Whilst not measured in the present study, physical activity levels are known to exert large influences on aerobic capacity and functional performance. Previous research in breast cancer patients has demonstrated that physical activity levels are significantly reduced following cancer diagnosis and during treatment $[26,27]$ and these reductions in physical activity negatively alter energy balance. We have recently reported that only $\sim 12 \%$ of Australian prostate cancer survivors are meeting sufficient exercise levels $(150 \mathrm{~min}$ of moderate intensity or 75 min of strenuous exercise per week and twice weekly resistance exercise) [28]. It is possible that in the current study physical activity levels were reduced following diagnosis and either they continued to decline as treatment time progressed or the side effects associated with declining physical activity levels did not present until later in treatment which may have contributed to the differences observed in aerobic fitness and RMR.

It was somewhat surprising that we observed no differences in the measured blood biomarkers or central blood pressure in relation to ADT exposure. With regard to the blood biomarkers, it may be that testosterone suppression has such a rapid effect, shown by the increased serum insulin levels previously reported by Dockery et al. [6] after only 3 months of ADT, which leads us to believe that these biomarkers may have stabilised within the first three months.

The strength of this study is that we directly measured $\mathrm{VO}_{2}$ during a maximum exercise stress test (gold standard assessment for aerobic capacity) rather than use of surrogate measures such as the 400 meters or $6 \mathrm{MWT}$ as well as 
assessments of RMR and central blood pressure. A limitation of this study is that patients were only assessed at a single time point during their treatment; hence, we were unable to continually monitor each participant as treatment time progressed. Further, the cross-sectional nature of the study does not permit us to infer causality; however, it provides initial evidence of decline in aerobic capacity measured objectively and it could be clinically meaningful to patients but needs further validation in prospective studies. However, it should be recognised that men in this study were volunteers for an exercise trial and therefore are not representative of all men with prostate cancer undergoing ADT. Lastly, given the exploratory nature of the study, we did not adjust for multiple comparisons [29] and, as a result, cannot discount the possibility that 1-2 of the significant differences may have been a chance finding.

\section{Conclusion}

In conclusion, we found that patients exposed to longer duration ADT had reduced cardiorespiratory capacity and RMR and these could have clinical meaningful implications. The exact mechanisms remain unclear as to why these cardiovascular parameters are further declining as the treatment time progresses and should be determined in future mechanistic studies. Intervention strategies to preserve cardiovascular capacity and RMR such as exercise medicine interventions have significant potential to counteract these forms of decline.

\section{Conflict of Interests}

The authors declare that they have no conflict of interests.

\section{Acknowledgments}

The data presented in this paper are from a randomized clinical trial funded by the National Health and Medical Research Council, Project Grant AppID 534409. Daniel A. Galvão received fund from the Cancer Council Western Australia Research Fellowship and Movember New Directions Development Award obtained through Prostate Cancer Foundation of Australia’s Research Program.

\section{References}

[1] G. W. Chodak, "Comparing treatments for localized prostate cancer-persisting uncertainty," The Journal of the American Medical Association, vol. 280, no. 11, pp. 1008-1010, 1998.

[2] M. R. Smith, J. S. Finkelstein, F. J. McGovern et al., "Changes in body composition during androgen deprivation therapy for prostate cancer," Journal of Clinical Endocrinology and Metabolism, vol. 87, no. 2, pp. 599-603, 2002.

[3] S. Basaria, D. C. Muller, M. A. Carducci, J. Egan, and A. S. Dobs, "Hyperglycemia and insulin resistance in men with prostate carcinoma who receive androgen-deprivation therapy," Cancer, vol. 106, no. 3, pp. 581-588, 2006.

[4] D. A. Galvão, N. A. Spry, D. R. Taaffe et al., "Changes in muscle, fat and bone mass after 36 weeks of maximal androgen blockade for prostate cancer," BJU International, vol. 102, no. 1, pp. 44-47, 2008.

[5] J. C. Smith, S. Bennett, L. M. Evans et al., "The effects of induced hypogonadism on arterial stiffness, body composition, and metabolic parameters in males with prostate cancer," Journal of Clinical Endocrinology and Metabolism, vol. 86, no. 9, pp. 42614267, 2001.

[6] F. Dockery, C. J. Bulpitt, S. Agarwal, M. Donaldson, and C. Rajkumar, "Testosterone suppression in men with prostate cancer leads to an increase in arterial stiffness and hyperinsulinaemia," Clinical Science, vol. 104, no. 2, pp. 195-201, 2003.

[7] S. M. H. Alibhai, H. Breunis, N. Timilshina et al., "Impact of androgen-deprivation therapy on physical function and quality of life in men with nonmetastatic prostate cancer," Journal of Clinical Oncology, vol. 28, no. 34, pp. 5038-5045, 2010.

[8] S. Basaria, "Androgen deprivation therapy, insulin resistance, and cardiovascular mortality: an inconvenient truth," Journal of Andrology, vol. 29, no. 5, pp. 534-539, 2008.

[9] N. L. Keating, A. J. O’Malley, and M. R. Smith, “Diabetes and cardiovascular disease during androgen deprivation therapy for prostate cancer," Journal of Clinical Oncology, vol. 24, no. 27, pp. 4448-4456, 2006.

[10] J. Myers, M. Prakash, V. Froelicher, D. Do, S. Partington, and J. Edwin Atwood, "Exercise capacity and mortality among men referred for exercise testing," The New England Journal of Medicine, vol. 346, no. 11, pp. 793-801, 2002.

[11] S. Gupta, A. Rohatgi, C. R. Ayers et al., "Cardiorespiratory fitness and classification of risk of cardiovascular disease mortality," Circulation, vol. 123, no. 13, pp. 1377-1383, 2011.

[12] D. A. Galvão, D. R. Taaffe, N. Spry, D. Joseph, and R. U. Newton, "Combined resistance and aerobic exercise program reverses muscle loss in men undergoing androgen suppression therapy for prostate cancer without bone metastases: a randomized controlled trial," Journal of Clinical Oncology, vol. 28, no. 2, pp. 340-347, 2010.

[13] R. U. Newton, D. R. Taaffe, N. Spry et al., "A phase III clinical trial of exercise modalities on treatment side-effects in men receiving therapy for prostate cancer," BMC Cancer, vol. 9, no. 1, article 210, 2009.

[14] W. R. Thompson, N. F. Gordon, and L. S. Pescatello, Eds., ACSM's Guidelines for Exercise Testing and Prescription, Lippincott Williams \& Wilkins, 8th edition, 2010.

[15] E. T. Howley, D. R. Bassett Jr., and H. G. Welch, "Criteria for maximal oxygen uptake: review and commentary," Medicine and Science in Sports and Exercise, vol. 27, no. 9, pp. 1292-1301, 1995.

[16] C. Compher, D. Frankenfield, N. Keim, and L. Roth-Yousey, "Best practice methods to apply to measurement of resting metabolic rate in adults: a systematic review," Journal of the American Dietetic Association, vol. 106, no. 6, pp. 881-903, 2006.

[17] E. O’Brien, F. Mee, N. Atkins, and M. Thomas, "Evaluation of three devices for self-measurement of blood pressure according to the revised British Hypertension Society Protocol: the Omron HEM-705CP, Philips HP5332, and Nissei DS-175," Blood Pressure Monitoring, vol. 1, no. 1, pp. 55-61, 1996.

[18] A. L. Pauca, M. F. O'Rourke, and N. D. Kon, "Prospective evaluation of a method for estimating ascending aortic pressure from the radial artery pressure waveform," Hypertension, vol. 38, no. 4, pp. 932-937, 2001.

[19] S. Nottin, G. Walther, A. Vinet et al., "Reproducibility of automated pulse wave velocity measurement during exercise. 
Running head: Pulse wave velocity during exercise," Archives des Maladies du Coeur et des Vaisseaux, vol. 99, no. 6, pp. 564-568, 2006.

[20] Expert Panel on Detectio, Evaluation, and Treatment of High Blood Cholesterol in Adults, "Executive summary of the third report of the National Cholesterol Education Program (NCEP) Expert panel on detection, evaluation, and treatment of high blood cholesterol in adults (Adult Treatment Panel III)," Journal of the American Medical Association, vol. 285, no. 19, pp. 24862497, 2001.

[21] B. A. Wall, D. A. Galvão, N. Fatehee et al., "Maximal exercise testing of men with prostate cancer being treated with ADT," Medicine and Science in Sports and Exercise, vol. 46, no. 12, pp. 2210-2215, 2014.

[22] C. E. Barlow, L. F. DeFina, N. B. Radford et al., "Cardiorespiratory fitness and long-term survival in 'low-risk' adults," Journal of the American Heart Association, vol. 1, no. 4, Article ID e001354, 2012.

[23] R. L. Weinsier, Y. Schutz, and D. Bracco, "Reexamination of the relationship of resting metabolic rate to fat-free mass and to the metabolically active components of fat-free mass in humans," The American Journal of Clinical Nutrition, vol. 55, no. 4, pp. 790-794, 1992.

[24] A. M. Johnstone, S. D. Murison, J. S. Duncan, K. A. Rance, and J. R. Speakman, "Factors influencing variation in basal metabolic rate include fat-free mass, fat mass, age, and circulating thyroxine but not sex, circulating leptin, or triiodothyronine," American Journal of Clinical Nutrition, vol. 82, no. 5, pp. 941948, 2005.

[25] M. Braga-Basaria, A. S. Dobs, D. C. Muller et al., "Metabolic syndrome in men with prostate cancer undergoing long-term androgen-deprivation therapy," Journal of Clinical Oncology, vol. 24, no. 24, pp. 3979-3983, 2006.

[26] M. L. Irwin, D. Crumley, A. McTiernan et al., "Physical activity levels before and after a diagnosis of breast carcinoma," Cancer, vol. 97, no. 7, pp. 1746-1757, 2003.

[27] W. Demark-Wahnefried, V. Hars, M. R. Conaway et al., "Reduced rates of metabolism and decreased physical activity in breast cancer patients receiving adjuvant chemotherapy," American Journal of Clinical Nutrition, vol. 65, no. 5, pp. 14951501, 1997.

[28] D. A. Galvão, R. U. Newton, R. A. Gardiner et al., "Compliance to exercise-oncology guidelines in prostate cancer survivors and associations with psychological distress, unmet supportive care needs, and quality of life," Psycho-Oncology, vol. 24, no. 10, pp. 1241-1249, 2015.

[29] K. J. Rothman, "No adjustments are needed for multiple comparisons," Epidemiology, vol. 1, no. 1, pp. 43-46, 1990. 


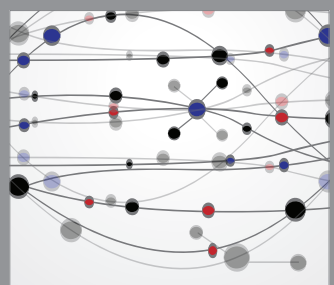

The Scientific World Journal
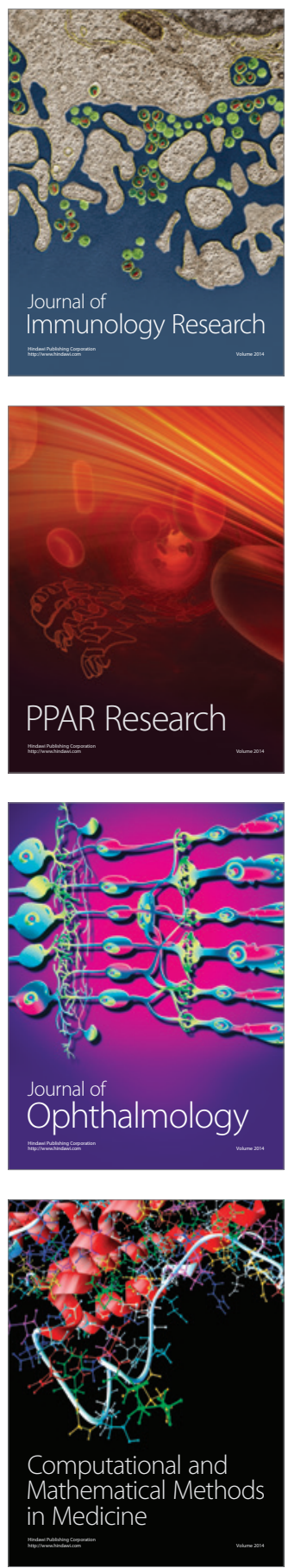

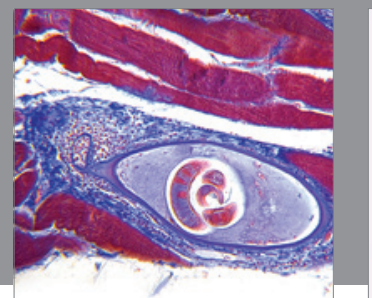

Gastroenterology

Research and Practice
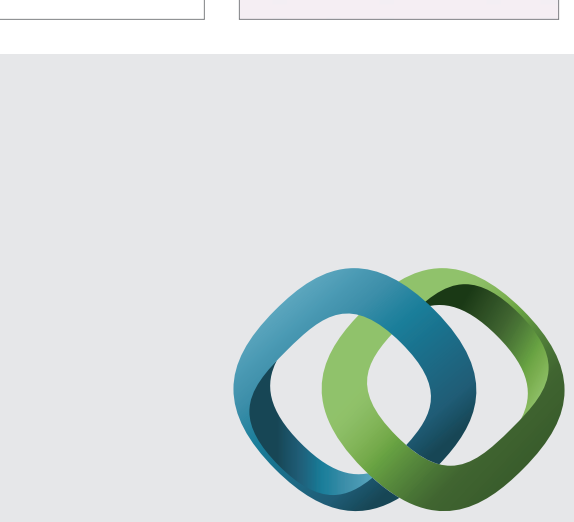

\section{Hindawi}

Submit your manuscripts at

http://www.hindawi.com
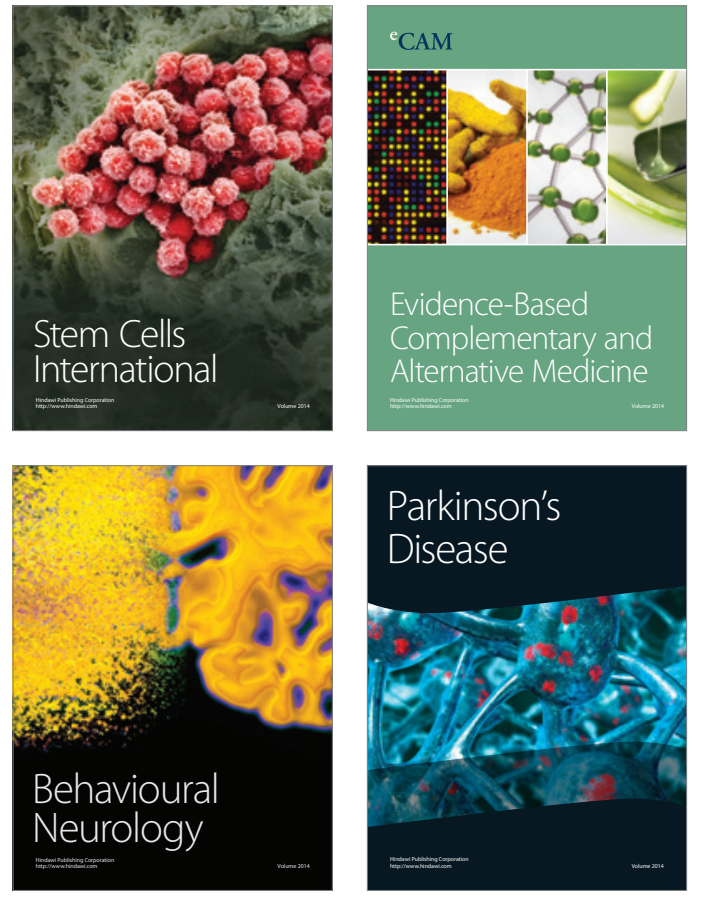
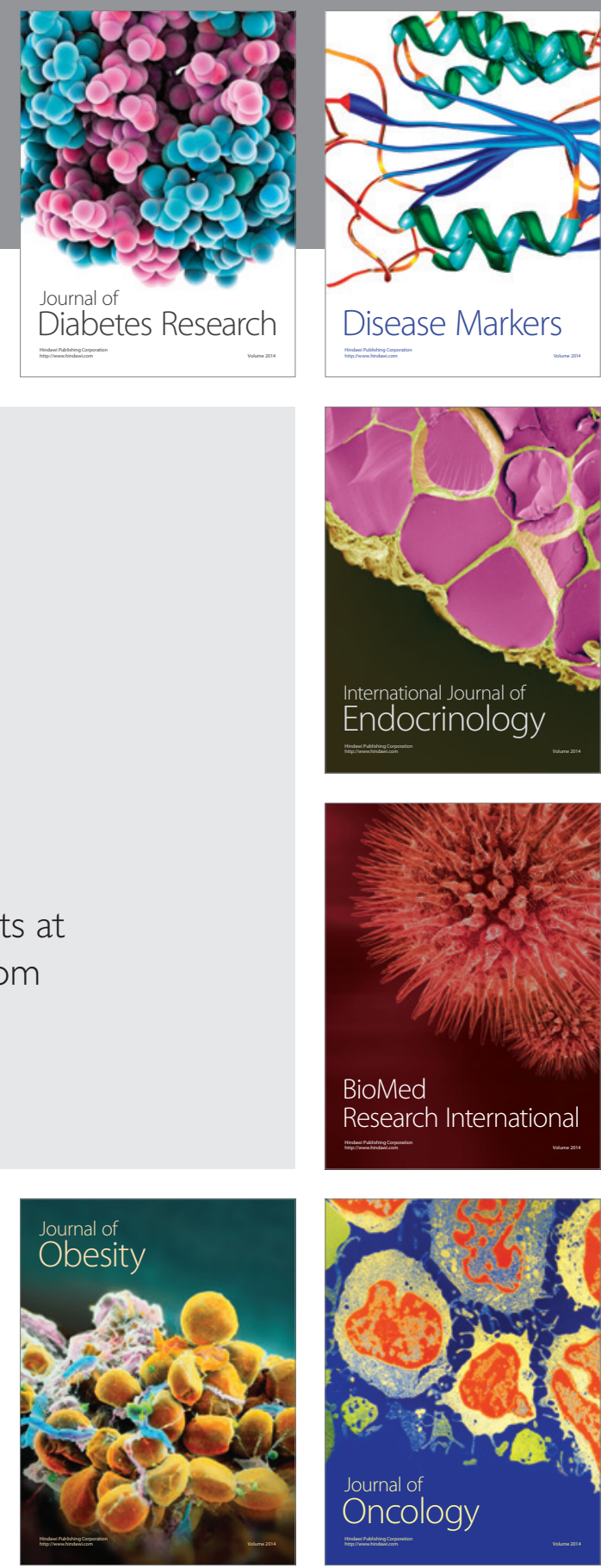

Disease Markers
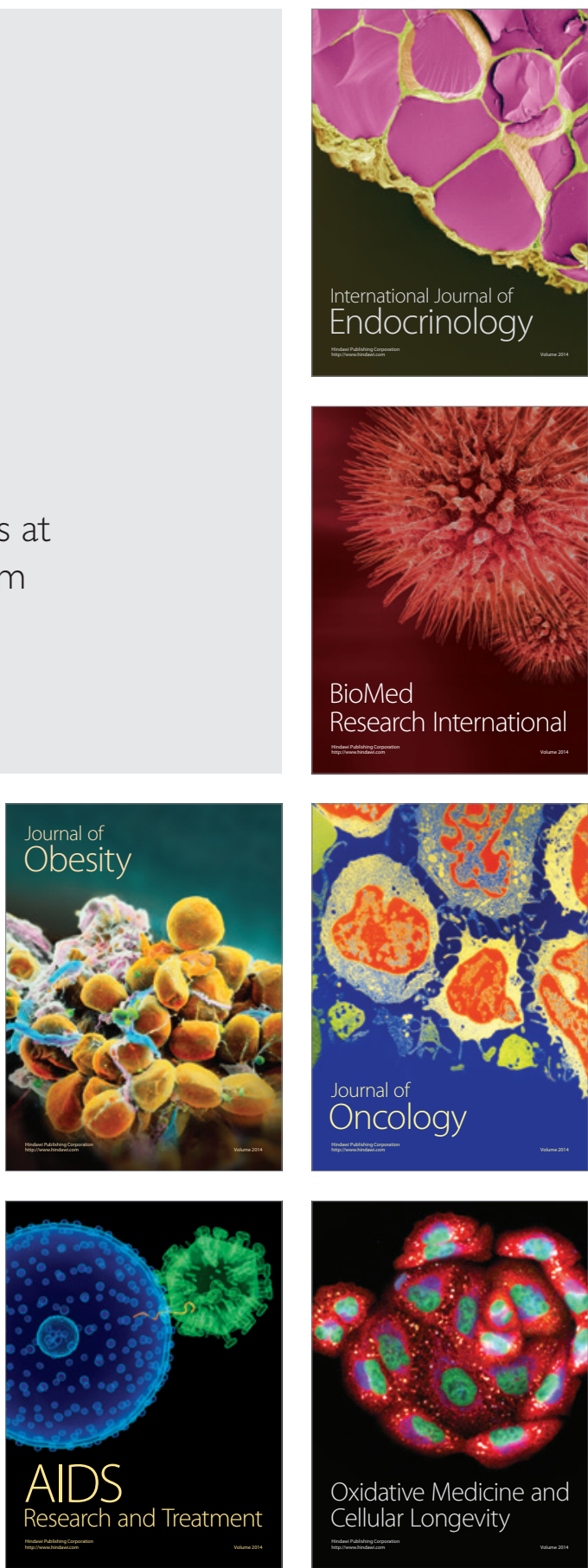